\title{
Corporeality of Architecture Experience
}

\author{
Katharina Voigt
}

Editorial Summary: In »Corporeality of Architecture Experience« Katharina Voigt examines the embodied knowledge in the perception and the exploration of architectural spaces. She highlights embodiment, experience, and sensation as primary fields of investigation. The interrelation of architecture and the human body is described as dependent on bodily ways of knowing and movement as access to sensory encounters with architecture. Relating to the practice of contemporary dance and particularly the work of Sasha Waltz, she regards the body as an archive, generator, and medium of pre-reflexive knowledge, emphasizing its resonance with the space. She exploits the potential which an investigation of the body-based, sensory experience holds when being explicitly addressed and regarded as an integrated part of both, the perception and the design of architecture. [Uta Graff]

Keywords: Corporeal Experience; Embodied Knowledge; Bodily Archive; Contemporary Dance; Sensorimotor Perception; Reciprocity of Space and Movement; Sensuality; Sasha Waltz \& Guests.

\section{Research Based upon Perception}

As architecture constitutes the built environment of the living world, it forms the context of our physical access to the world of matter. The process of creation and anticipation in architectural design and the actual exploration of architectural situations are equally dependent on past and present lived experiences. Setting perception at the core of research in architecture, the corporeality of the perceiver becomes an inevitable concern of investigation: Spatial perception is an enactive process of movement, exploration, sensual effects, and the sensorimotor encounter of the individual with the physical realm. The perception of space is bound to the body and is preconditioned by both the self-awareness of the body and the perceptual sensation through the body. Therefore, body consciousness and the corporeal outreach into the world condition and direct spatial perception. As according to Maurice Merleau-Ponty, »my whole body for me is not an assemblage of organs juxtaposed in space. I am in undivided possession of it and I know 
where each of my limbs is through a body image in which all are included « (Merleau-Ponty 2003/1962 [1945]:112-113). Proprioception is a given condition of our embodied existence. Accordingly, the investigation of the perception of space and architecture is inseparably linked to the body, as it is its indispensable prerequisite. The more differentiated the sensorimotor capacity of the body and the corporeality of movement in space are exploited, the more precise the knowledge of how we perceive architecture can be gained:

»If bodily space and external space form a practical system, the first being the background against which the object as the goal of our action may stand out or the void in front of which it may come to light, it is clearly in action that the spatiality of our body is brought into being, and an analysis of one's own movement should enable us to arrive at the better understanding of it. By considering the body in movement, we can see how it inhabits space and, moreover, time, because movement is not limited to submitting passively to space and time, it actively assumes them [...].« (Merleau-Ponty 2003/1962 [1945]: 117)

Against the background of perception, research in architecture concerns the interrelation between space and body, based upon their respective feasibility to resonate with one another and the reciprocity of their interaction. The choreographic techniques and procedures in contemporary dance serve as tools for an in-depth understanding of the corporeal effect that architectural spaces have on the human body. They enhance the phenomenological horizon of architectural space and the corporeality of the body as a connection to the world of the living. The sensual dimension of architecture experience, as well as its impact on the corporeality of its perceiver, becomes therefore intelligible and attainable to be included in the process of architectural design.

\section{Architectural Encounter as Corporeal Experience}

This research pursues the question of how to find a deeper understanding of the corporeal impact architectural spaces have on the human body. Contemporary dance has the potential to serve as an effective technique to increase corporeal awareness and body-knowledge. In this respect, it is crucial to inquire how it can be made accessible as a tool to comprehend the sensory and corporeal agency of architectural spaces and the anticipation of their perception in the architectural design. 
Theoretical and experiential examinations of the reciprocal interaction of architecture and corporeal spatial perception form the objective of this research. For a procedural and multisensory experience of space, the corporeality of the perceiver appears to be an indispensable prerequisite, as $»$ there would be no space for me at all, if I had no body « (Merleau-Ponty 2003/1962 [1945]: 117). Moreover, this space only emerges to me when I conduct movement within it or refer to the memory of prior sensorimotor experience. Contemporary dance is a rich source to foster knowledge and understanding of the interdependence of body and space:

»As a medium, dance needs the body, but its true media are space and time. Space and time are articulated for human beings by the medium of the body. Dance literally embodies space and time or rather the unity of space and time.« (Weibel 2014: 11)

In order to transition the bodily knowledge from contemporary dance into the discipline and discourse of architecture, the role of the body in the architectural realm needs to be specifically addressed. Key questions regarding the embodied dimension of perception and experience in architectural research are: How is the sensual and sensorimotor experience of architectural spaces constituted? How can the associative and tacit body knowledge of (previous) spatial explorations be made accessible for the architectural design? How can contemporary dance practices contribute to the anticipation and integration of future embodied, sensorimotor architecture experiences in the design process?

\section{Hypothesis}

In reference to the last question, the hypothesis of this research claims: The immanent knowledge of the body enables the anticipation of future architectural experience in the design process. Contemporary dance - and in particular the choreographic work and oeuvre of Sasha Waltz - exemplifies an attempt to make this tacit embodied knowledge explicit and available as a design tool. The tacit body-knowledge we bear in our memory from prior architectural encounters and tangible spatial contexts can be made available and accessible for the anticipation of architectural perception in the design process if we conceive the body as a medium and integrate body-based working methods into the discipline of architecture. 


\section{Methodologies, Approaches and Alignments}

This research project is essentially divided into three sections: the theoretical framework of phenomenology and perception theory, the examination of Sasha Waltz's working methods and oeuvre as a case study, and the transfer knowledge from contemporary dance as a constituent component of the architectural design.

Phenomenological theory and concepts concerning the interrelation of body, movement, and space are reflected. The French movement in phenomenology is specifically referred to, as - opposed to its German counterpart it accentuates fluid and motion-dependent interrelations between the individual and the living world, advocating an enactive and embodied approach to their reciprocal interconnection. Furthermore, recent findings from cognitive sciences, philosophy of embodiment, arts theory, and performance studies frame the project's theoretical horizon.

Sasha Waltz's oeuvre serves as an artistic exemplum to substantiate the theoretical considerations. Waltz's working method and selected works of hers with explicit relation to space are examined in regard to their adaptation of architectural spaces, both in-situ and on stage. The respective spatiality of their repertory of gesture and movement is explored correspondingly. Waltz's procedures of creation and her working methods are specifically addressed to extract a superordinate understanding of her choreographic investigation of space. Her work is examined with a particular emphasis on its emerging relationship with space. In addition, her genuine choreographic techniques are compared with design methods in architecture, to which they have a close affinity, as both draw from the visual arts, using drawings and sketches in the conception and to develop ideas. While the experiential, sensual, and corporeal knowledge of architectural spaces emanates from the means of contemporary dance and the work of Sasha Waltz \& Guests, this research aims to reach further, incorporating the practices of contemporary dance and their respective embodied knowledge into the process of architectural design.

\section{Aims and Objectives}

Despite the transdisciplinary nature of this research project, the investigation is led with the expertise and from the perspective of an architect. The overarching aim is to consider the dynamic and enacting potential of 
architecture and the built environment and to integrate embodied knowledge and corporeal memory into the anticipation of future architectural experiences in the design process. Working methods and procedural techniques from contemporary dance are applied to architecture, seeking a profound understanding of sensorimotor perception. Thus, it is intended to align the spatial experience with an architecture that is conceived, designed, and created as an experiential space.

\section{Embodiment, Experience, and Sensation}

Research in architecture in regard to its perception depends upon a changing understanding of architecture itself. As it is regularly considered to constitute the matter framework of the man-made built environment - which serves as a vessel containing human action, adaption, and appropriation its investigation under the premise of perception takes more elusive, immaterial phenomena into account. Considering the embodied, lived experience, and sensual effects of architectural spaces, the concept of architecture as the manifest world of solitary objects falters and is replaced by the subjectivity of the individual experience. Hence, the seemingly objective access to the architectural context becomes questionable.

Experience does not appear as a singular momentary incident, but is integrated into a superordinate context of individual memories, associations, and imaginations. The present sensations and perceptions evoked by an encounter with a specific space are interwoven with a lively and everchanging assemblage of fragments from previous experiences. They correlate in imaginary projections, so that perceiving emulates an inventive and creative act of mental associations. "People say that life takes place. It is architects who make place. The architect's role is to give existence, or presence to the spaces that are the acts of our lives and the foundation of our memories. The very nature of such interactions with the world forms culture, cities, and dwelling " (Condia 2019: 183). Therefore, the architectural context is to be considered as an invitation for exploration and interaction, rather than the condition for adaption and appropriation, as it is considered in a static, vessel-like understanding of architecture. In this sense, architecture and its perceivers appear to be mutually dependent, as an interresponsive resonance is evoked between the two.

According to Harry Francis Mallgrave, this lively interaction draws reference to the philosophical term of the Vita Activa, which »in this sense entails 
not only making things but making experiences, which is the hallmark of our vital natures" (Mallgrave 2018: 130). This literal activation of architectural perception exposes the corporeality of the body as its medium:

»The existentially most important knowledge of our everyday life [...] does not reside in detached theories and explanations, but in a silent knowledge that lied beyond the threshold of consciousness fused with the daily environmental and behavioural situations. [...] We experience a work of art or architecture through our embodied existence and identification.« (Pallasmaa 2001: 14-16)

The body-knowledge contributes to the conscious memory of the mind, as accordingly, »the experienced, remembered, and imagined are qualitatively equal experiences in our consciousness", which is evident in the fact that »we may be equally moved by something evoked by the imagination as by the actually encountered« (Pallasmaa 2001: 18-20). Through the inscribed traces of previous experiences, the body becomes a lively archive that preserves past perceptions, integrates present sensations, and - against the background of this accumulated knowledge - constitutes the anticipation of future experience. It forms a bodily archive of experiences from encountered spatial situations. Through a deepened awareness of this corporeal repertory of pre-noetic memories, our bodies have the potential to become tools of anticipation. Architectural design requires preconceived knowledge to imagine its abstract conception on a one-to-one scale. Through our capacity to resonate and to empathize with our surrounding world, we are able to imagine its experiential appearance. The reference to prior lived experience, contained in our bodily memory, enables us to read architectural plans and to imagine their respective spatial qualities. Furthermore, the enactive attempt at a physical model - the sensual touch of its surfaces or moving about this scaled object of the intended spatial sequence - triggers a corporeal interaction, inviting the spectator to take up a position in relation to the object.

Taking into account the practice, work, and oeuvre of Sasha Waltz requires the personal archive of her spatial memory as well as her contribution to the constitution of future spatial and corporeal encounter. For Waltz, dance inevitably relates to space - on one hand in etymological sense of choreography as a corporeal engraving of space, and on the other hand in the sense in regard to the actual space within which the dance takes place 
and where certain aspects of an architecture are revealed by the means of dance. "Spaces, says Sasha Waltz, are the basis of her work, the starting point, the beginning (Schlagenwerth 2008: 7); spatial gestures and figures of movement are inseparably interconnected and affect each other as interrelating counterparts. This intertwining between body and architecture, expansion and compression, is essential for the anticipation during the conceptual process. Unless we evoke actual experience, the architectural concept remains an abstract approximation outside of reality. A holistic attempt at architectural design requires the integration of its phenomenological qualities and its impetus for movement, interaction, and adaption as intrinsic aspects of its conceptual creation.

\section{Conclusion}

Often, the interrelation of architectural spaces and the human body remains unnoticed, as it affects us in a pre-noetic manner. Beyond our conscious recognition, it might even remain unaware. Despite the all-encompassing sensual experience of actual architectural encounter, it is a demanding challenge to highlight its constitutive parts in order to make them accessible for the design process. According to Alban Janson and Florian Tigges, spatial experience is an interplay between corporeal expansion and the impact of space on the body, as upon entering a space »our personal sphere, seeks to engage with the space and we become aware of »how it expands either hesitantly or instantaneously, taking in the space and feeling it out or filling it up in its various directions and forms through the spatial extension of our own subjectivity.« (Janson/Tigges 2014 [2013]: 107).

In regard to the conceptual creation of architectural designs, this very impression of an actual encounter remains the most challenging to address. "As we enter the space, the space enters us, and the experience is essentially an exchange and fusion of the object and the subject«; Pallasmaa describes the all-encompassing effect that architectural encounters have on their perceivers (Pallasmaa 2014: 232). Vice-versa, "as the space enters us, we enter the space « as anticipation in the design process. This mutual condition illustrates the involvement of lived experience and corporeal encounter in actual architectural space, such as in its anticipation in the design process. Moreover, the intersubjectivity of experience in general and the distinct subjective, individual impression finds a nuanced differentiation in Pallasmaa's observation: As the individual object blurs to form the 
totality of an architectural expression, its perception includes both an individual, subjective attempt and an intersubjective, generic sensual encounter. In this sense, the body appears to be an "archive in motion «, an assemblage of lived experiences, embodied memory and associations (cf. Wehren 2016). Integrating the methodologies from contemporary dance and the work of Sasha Waltz into architectural research reveals the resemblance between the two disciplines. Procedural analogies become evident as commonalities; Waltz's in-situ Dialogue series exploits architecture's potential as the impulse to generate the movement figures inscribed into the space, whereas her productions rather highlight the reciprocal interrelation between the spatiality of the body and the corporeality of architecture. The adaptation of different locations in Berlin as venues and working spaces, has an influence on the development of the work. Each of the interim uses provides valuable impulses for the development of Sasha Waltz's work. By working in the respective spaces and elaborating site-specific performances for each venue, these places are closely linked to the development of the work.

Analogies between the figures of movement conducted in a particular space and the configurations and gestures initiated by the space itself are essential for the consideration of architecture as a realm of corporeal experience: One's own body is drawn upon as a medium of architectural experience. Aiming for corporeal access to the experience of architecture, we are eager to develop architectural designs that elicit the nuanced sensations of an actual experience already during the development process. Experiences of architecture indispensably depend upon an attentive body. As the transdisciplinary investigation of contemporary dance and architecture grants access to the body as a medium to experience space, it makes the tacit, pre-reflexive, body-based knowledge explicit and adaptable to the design. With the pronounced emphasis on Waltz's handling of architectural spaces, the procedural, multisensory, and sensorimotor dimensions of architectural perception are fostered. It is the aim of this research to exploit the potential held by the human body to experience the sensory aspects of architecture including its memory, envisioning, and anticipation - in order to include its capacities and genuine knowledge in the repertoire of established cultural techniques of architectural design, such as sketching, drawing, model making, visualization or description. 


\section{References}

Condia, Bob (2019): »What Architects Do: Construct Culture«, in: Alessandro Gattara/Sarah Robinson/Davide Ruzzon (eds.), Intertwining, Baukultur, 02/2019, Mimesis International.

Mallgrave, Harry Francis (2018): From Object to Experience: The New Culture of Architectural Design, London: Bloomsbury Publishing.

Merleau-Ponty, Maurice [1945]:

Phénoménologie de la perception. - English translation: Phenomenology of Perception, transl. by Colin Smith (2003 [1962]), London/New York: Routledge \& Kegan Paul.

Pallasmaa, Juhani (2001): »Lived Space. Embodied Experience and Sensory Thought«, in: The Visible and the Invisible, OASE (58), Amsterdam: Sun Publishers, 13-34.
Pallasmaa, Juhani (2014): »Space, Place and Atmosphere. Emotion and Peripheral Perception in Architectural Experience«, in: Lebenswelt: Aesthetics and Philosophy of Experience, 230-245. doi: $10.13130 / 2240-9599 / 4202$

Schlagenwerth, Michaela (2008): Nahaufnahme Sasha Waltz. Gespräche mit Michaela Schlagenwerth, Berlin: Alexander Verlag.

Weibel, Peter (2014): »Between >Performative Turn< and >Installative Turn«, in: Christiane Riedel/Yoreme Waltz/Peter Weibel (eds.), Sasha Waltz. Installations, Objects, Performances. Ostfildern: Hatje Cantz.

Wehren, Julia (2016): Körper als Archiv in Bewegung. Choreographie als historiographische Praxis, Bielefeld: transcript Verlag. 
